\title{
The Effect of the Rolling Direction, Temperature, and Etching Time on the Photochemical Machining of Monel 400 Microchannels
}

\author{
Deepakkumar H. Patil and Sadaiah Mudigonda \\ Department of Mechanical Engineering, Dr. Babasaheb Ambedkar Technological University, Vidyavihar, Lonere, \\ Raigad, Maharashtra 402-103, India \\ Correspondence should be addressed to Deepakkumar H. Patil; dhpatil@dbatu.ac.in
}

Received 14 April 2016; Accepted 27 July 2016

Academic Editor: Belal F. Yousif

Copyright (C) 2016 D. H. Patil and S. Mudigonda. This is an open access article distributed under the Creative Commons Attribution License, which permits unrestricted use, distribution, and reproduction in any medium, provided the original work is properly cited.

\begin{abstract}
The present paper describes the effect of the rolling direction on the quality of microchannels manufactured using photochemical machining (PCM) of Monel 400. Experiments were carried out to fabricate microchannels along and across the rolling direction to investigate the effect of the grain orientation on microchannel etching. The input parameters considered were channel width and rolling direction, whereas the depth of etch was the response parameters. Different channels of widths of 60, 100, 150, 200, and $250 \mu \mathrm{m}$ were etched. The effects of the etching time and temperature of the etchant solution on the undercut and depth of the microchannels were studied. For good quality microchannels, the effects of spinning time, spinning speed, exposure time, and photoresist film strength were also taken into consideration. Optimized values of the above were used for the experimentation. The results show that the depth of etch of the microchannel increases more along the rolling direction than across the rolling direction. The channel width and depth are significantly affected by the etching time and temperature. The proposed study reports an improvement in the quality of microchannels produced using PCM.
\end{abstract}

\section{Introduction}

In recent years, microprecision machining has received much attention because of the demands of miniaturization in the manufacturing and process industries for technological applications such as medical devices, biotechnology, microreactors, labs-on-a-chip, electronic components, optical devices, communication technologies, and precision engineering [1-4]. When employing conventional machining techniques, inherent problems of tool wear, heat generation at the chiptool contact, and the relative difficulty of machining threedimensional microparts have to be factored in. The machining process increases the product cost because of the involvement of the tooling cost, maintenance cost, and running cost. In comparison, chemical machining is low cost and causes no chemical or thermal stresses [5].

Photochemical machining is one of the most important lithographic techniques. Complex parts can be manufactured using accurate deposition and exposure of a photoresist pattern followed by etching. Photochemical machining has matured rapidly, and its ability to resolve ever-smaller features is constantly improving. For microfabrication, this continued improvement in the photochemical machining resolution has impeded the adaptation of alternative, higherresolution lithographic techniques, such as X-ray lithography. Research during the past 10 years on the demands of microfabrication has increased drastically. The manufacturing of miniaturized high-aspect ratio components is a need of today's era.

Generally, micromachining, laser machining, dry machining, and electrochemical machining have been used for the fabrication of microchannels [6]. He et al. fabricated crossconnected microchannels using multicutter milling [7]. PCM is a good alternative for high-cost nonconventional machining processes. PCM does not need high investment and is a cheaper process for micromachining operations. Being a chemical etching process, it damages metal but can be an efficient method for converting metal into useful products [8]. To accomplish all of the above needs, studying the effect of the rolling direction on the miniaturized features plays a vital 
role in the PCM process. From the literature, it is clear that no research has reported on the effect of the rolling direction in the PCM of microfeature components.

The rolling direction greatly affects the quality of the product. In this work, the fabrication of microchannels is carried out. During the testing and analysis, it was found that there is a considerable effect of the rolling direction on microchannel accuracy. Numerous works have already been carried out in this area, but in the field of photochemical machining, the rolling direction effect has yet to be studied. Ha and Park [9] studied the effect of the rolling direction on the development of the microstructure, texture, and mechanical properties of AZ31B alloys. Topic et al. observed the influence of the rolling direction on the strength and ductility of aluminium and aluminium alloys produced by accumulative roll bonding [10]. The relationship of the microstructure and texture with the rolling direction in coldrolled metastable austenitic steel was investigated by Park et al. [11]. It was shown that under unidirectional rolling the laths of $\alpha$ martensite that were produced were uniformly thinner, with an increasing thickness reduction. On the other hand, under the cross-rolling condition, the resulting martensite laths varied in width and exhibited irregular bends. Kogut [12] investigated the effect of the direction of rolling on the crack resistance of bridge steels. It was noted that among the investigated steels the highest resistance to brittle fracture was shown by 15 G2AFDps steel when the crack is parallel to the direction of rolling.

Monel 400 was chosen as a specimen for the present study. Monel 400 has superior mechanical and thermal stability and can withstand a wide variety of severe operating conditions, such as corrosive environments and high stress [13]. Monel 400 also exhibits resistance to cavitation erosion. It can work in all types of atmospheres, including nuclear fuel reprocessing reactors. Monel 400 works excellently where reboiling conditions exist and is best suited for heat exchanger applications [14, 15]. Monel 400 has less than one-half the erosion rate of nickel in industrial atmospheres and has found extensive commercial use [16].

The photoresist coating thickness also affects the accuracy of the component. The photoresist temperature and the spinning rate are the most significant factors in the photoresist coating process [17]. The substrate can be coated by using either the spin coating or deep coating method. Obtaining a uniform photoresist coating depends on using a high spin rate and the viscosity of the photoresist [18]. SU-8 photoresist is extensively used in the manufacture of microelectromechanical devices $[19,20]$, but the cost of SU-8 photoresist is higher than that of LPR 1020 photoresist.

\section{Experimental Details}

The film strip phototool that was used in the experimental study had five different slot widths, as shown in Figure 1: 250, $200,150,100$, and $60 \mu \mathrm{m}$. A $4800 \mathrm{dpi}$ offset printer was used to print the phototool. The specimens were cut to the size of $30 \mathrm{~mm} \times 30 \mathrm{~mm}$ on a sheet of thickness $0.1 \mathrm{~mm}$. The initial roughness $\left(R_{a}\right)$ of the substrate was $183 \mathrm{~nm}$, as measured with
TABLE 1: Composition of Monel 400.

\begin{tabular}{lccccccc}
\hline Element & $\mathrm{C}$ & $\mathrm{Fe}$ & $\mathrm{Mg}$ & $\mathrm{Ni}$ & $\mathrm{S}$ & $\mathrm{Si}$ & $\mathrm{Cu}$ \\
\hline \% wt & 0.3 & 2.5 & 2.0 & 63 & 0.024 & 0.5 & 32 \\
\hline
\end{tabular}

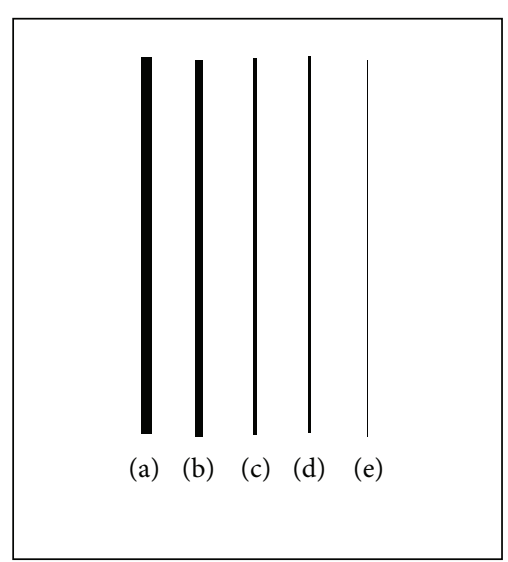

FIGURE 1: Phototool of different slot widths: (a) $250 \mu \mathrm{m}$; (b) $200 \mu \mathrm{m}$; (c) $150 \mu \mathrm{m}$; (d) $100 \mu \mathrm{m}$; and (e) $60 \mu \mathrm{m}$.

a Taylor Hobson roughness tester. The composition of Monel 400 is shown in Table 1.

Before coating with photoresist, all specimens were cleaned using an ultrasonic cleaner. Cleaning was carried out at $80^{\circ} \mathrm{C}$ for $50 \mathrm{~min}$. After the ultrasonic cleaning, all of the specimens were rinsed using dry air. Then, the specimens were coated with LPR 1020 liquid film photoresist (negative type) using a spin coater (Spectron-India). Specimens were coated at different spinning speeds to achieve specific coating thicknesses. The coating thickness was measured using a contact-type thickness gauge (Mextech CM 8823). Soft baking was carried out on the coated photoresist in a furnace at $100^{\circ} \mathrm{C}$ for $10 \mathrm{~min}$. Soft baking helps to adhere the photoresist on the substrate. Ultraviolet (UV) exposure was carried out using UV tubes of $365 \mathrm{~nm}$ wavelength on an exposure unit (Phonix make). Postexposure baking was used to completely dry the photoresist film. The temperature and baking time of the photoresist film were $100^{\circ} \mathrm{C}$ and $10 \mathrm{~min}$, respectively. Hard baking is performed after the development of the substrate to minimize outgassing [21] and improves the thermal and physical stability. Hard baking was performed at $120^{\circ} \mathrm{C}$ for $10 \mathrm{~min}$.

Experiments were conducted to find the correct exposure time and exposure dose. The exposure dose $(c)$ is calculated by the equation $I \times t=c$, where $I$ is the intensity of the light source and $t$ is the time of exposure [22]. Overexposure and underexposure can cause different line widths [23]. The exposure time for the proper polymerization of the photoresist depends on the thickness of the resist, the coating type, and the light source intensity. The light intensity was constant at 4866 lux. Seven levels of exposure time, namely, 30, 60, 90, $120,150,180$, and $210 \mathrm{~s}$, were used. The correct exposure time and exposure dose were established by measuring the line width under a Nikon microscope. A scratch tester (Ducom 


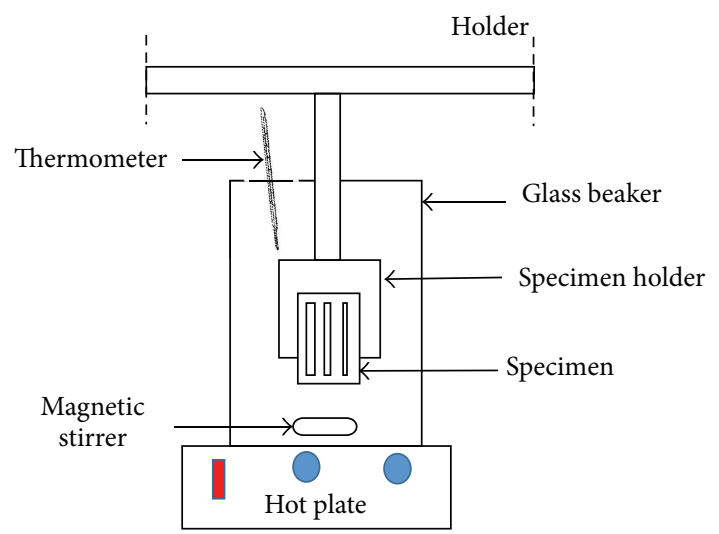

FIgURE 2: Experimental setup for Monel 400 etching.

India) was used to check the peel-off resistance of the photoresist.

The exposed specimens were developed for 40 to $70 \mathrm{~s}$ and subsequently rinsed with clean water. The unexposed area of the photoresist was dissolved in the developer. After the development of the photoresist, the film was washed with distilled water to wash out residual developer solution and dried using filter paper. The process from the photoresist coating to washing out the developer is called the microlithography process [21].

Experiments were conducted using one factor at a time, using $500 \mathrm{~g} / \mathrm{L}$ concentrations of etchant, at a constant temperature of $50^{\circ} \mathrm{C}$, and $5 \mathrm{~min}$ etching time as fixed parameters for evaluating the effect of the rolling direction. Experiments on the effect of the etching time and temperature of the etchant solution on the width and depth of the microchannels were also studied. The temperature levels were 50, 55, 60, 65, and $75^{\circ} \mathrm{C}$, and the etching time levels were $3,4,5,6$, and $7 \mathrm{~min}$. The experimental setup for Monel 400 etching is shown in Figure 2.

\section{Results and Discussion}

Monel 400 is an alloy that contains approximately $63 \%$ nickel and $32 \%$ copper. The addition of copper enhances the resistance of nickel to nonoxidizing acids [24].

The material removal in this process is due to the chemical reaction of nickel and copper with $\mathrm{FeCl}_{3}$. The basic chemistry (mechanism) behind the material removal is explained below:

$$
\begin{gathered}
\mathrm{FeCl}_{3}+\mathrm{Cu} \longrightarrow \mathrm{FeCl}_{2}+\mathrm{CuCl} \\
\mathrm{FeCl}_{3}+\mathrm{CuCl} \longrightarrow \mathrm{FeCl}_{2}+\mathrm{CuCl}_{2}
\end{gathered}
$$

Copper has an electronic configuration (Ar) $3 d^{10}, 4 s^{1}$. Here, copper has the " $1 \mathrm{~s}$ " orbital electron on top filled. The " $\mathrm{d}$ " shell electrons are characterized by high ductility and electrical conductivity. The filled outermost " $d$ " shell does not contribute to interatomic interactions, which is dominated by " $\mathrm{s}$ " shell electrons through metallic bonding. Iron and copper are two dissimilar metals with incomplete " $d$ " shells. The metallic bond in copper is lacking in covalent character and is relatively weak. Therefore, sharing of the electrons becomes easy. Chloride also gives off heat in an exothermic reaction, which increases the rate of reaction. Here, chloride undergoes a hydrolysis reaction.

$\mathrm{Ni}$ has an electronic configuration (Ar) $4 \mathrm{~s}^{2}, 3 \mathrm{~d}^{8}$. Ni has a " $2 s$ " orbital that is completely filled. The completely filled " $\mathrm{d}$ " shell element does not contribute to interatomic interactions, which is dominated by " $\mathrm{s}$ " shell and " $\mathrm{d}$ " shell electrons. Covalent bonds form easily due to the filled "s" orbital and " $d$ " orbital and also due to the lesser sharing of electrons. Due to the above reasons, the rate of reaction increases in $\mathrm{Ni}$, but it is less than that of copper. The corresponding reaction is given below:

$$
\mathrm{FeCl}_{3}+\mathrm{Ni} \longrightarrow \mathrm{NiCl}_{3}+\mathrm{Fe}
$$

A comparison of the relative oxidizing and reducing capabilities is explained below:

$$
\begin{aligned}
\mathrm{FeCl}_{3} & \longrightarrow \mathrm{Fe}^{3+}+3 \mathrm{e}^{-} \\
\mathrm{Cu}^{2+}+2 \mathrm{e}^{-} & \longrightarrow \mathrm{Cu} \\
\mathrm{Ni}^{2+}+2 \mathrm{e}^{-} & \longrightarrow \mathrm{Ni}
\end{aligned}
$$

Copper and nickel have oxidizing power, that is, the capacity to lose electrons, and Fe has reducing power, that is, to gain electrons. Therefore, the reaction of $\mathrm{Cu}$ and $\mathrm{Ni}$ with Fe takes place easily. The greater the reduction potential, the easier the reduction of ions. Therefore, the oxidation and reduction processes become faster and help increase the reaction rate.

The following reactions show that $\mathrm{Cu}$ and $\mathrm{Ni}$ have greater reduction potentials than Fe:

$$
\begin{aligned}
& \mathrm{Cu}^{2+}+2 \mathrm{e}^{-} \longrightarrow \mathrm{Cu}=0.16 \text { volt potential } \\
& \mathrm{Ni}^{2+}+2 \mathrm{e}^{-} \longrightarrow \mathrm{Ni}=-0.23 \text { volt potential } \\
& \mathrm{Fe}^{2+}+2 \mathrm{e}^{-} \longrightarrow \mathrm{Fe}=-0.41 \text { volt potential }
\end{aligned}
$$

The greater the oxidation potential of a metal, the stronger its capacity to lose electrons and the greater its electron mobility and reactivity, which makes the rate of reaction faster.

\section{Effect of Spinning Speed and Spinning Time on Photoresist Film Thickness}

Figure 3(a) shows the effect of the photoresist film thickness on the spinning speed where the photoresist patterns have been developed completely. It has been observed that an increase in spinning speed causes the film thickness of the photoresist to decrease because of centrifugal force. Usually, the pattern resolution of the photoresist film depends on the film thickness [23]. The pattern resolution is improved with a thin photoresist film, but the etching ability will be affected, as shown in Figure 3(b).

Figure 3(c) shows the effect of the spinning time on the photoresist film thickness. When the spinning time increases, the photoresist thickness decreases due to the 


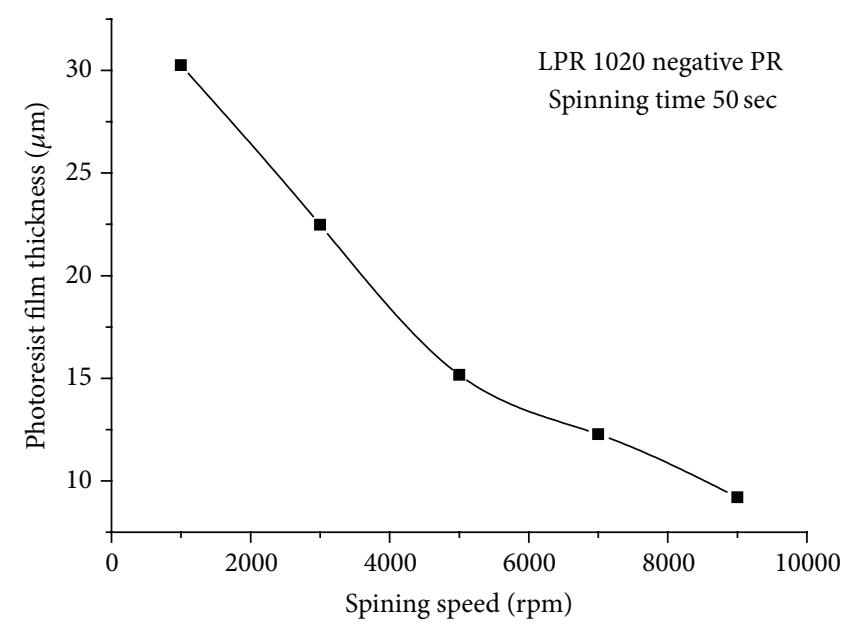

(a)

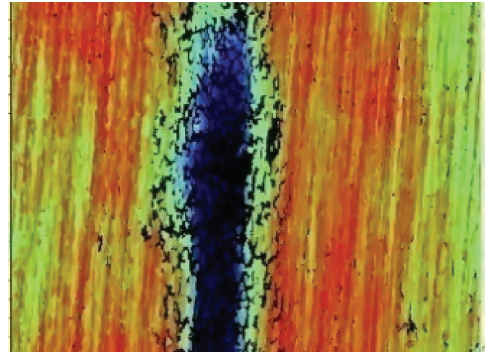

(i)

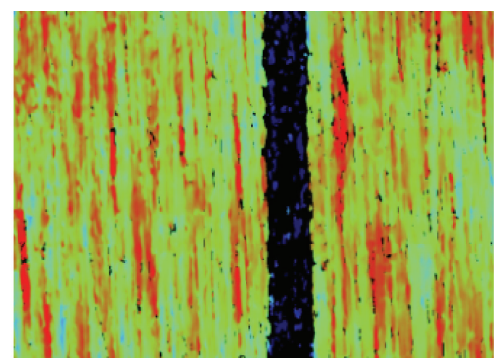

(ii)

(b)

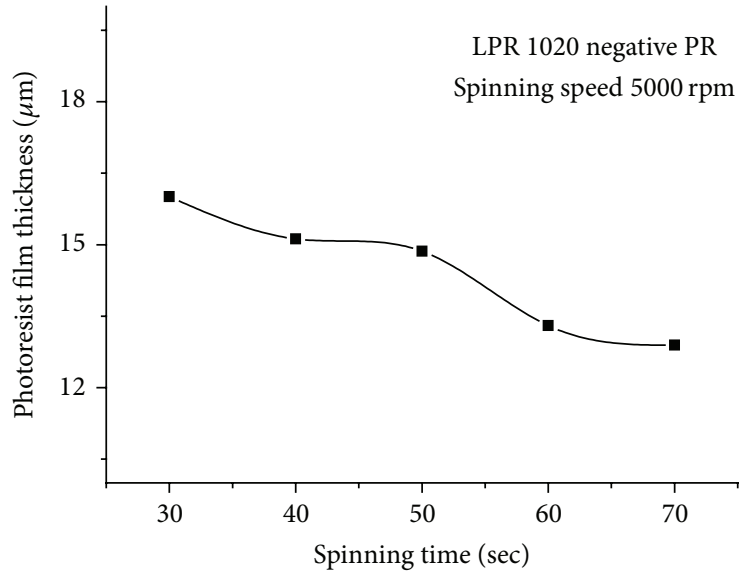

(c)

Figure 3: (a) Effect of spinning speed on photoresist film thickness for a spinning time of $50 \mathrm{~s}$. (b) Effect of photoresist coating on etching of micro channels. (i) Substrate with photoresist film thickness of $9.20 \mu \mathrm{m}$; (ii) substrate with photoresist film thickness of $15.16 \mu \mathrm{m}$. (c) Effect of spinning time on photoresist film thickness for a spinning speed of $5000 \mathrm{rpm}$.

evaporation of solvent, causing more time to be required to spread out the photoresist in the radial direction of the substrate. Figure 3(c) shows that a 30 s spinning time achieves a $16.01 \mu \mathrm{m}$ photoresist thickness, and it decreases to 15.12 , $14.86,13.3$, and $12.89 \mu \mathrm{m}$ for time intervals of $40,50,60$, and $70 \mathrm{~s}$, respectively. The peeling off of the photoresist took place during the etching for the lower photoresist thickness.

\section{Effect of Exposure Time on Image Width and Photoresist Strength}

Figure 4 and Table 2 show the effect of the exposure time on the line width and strength of the photoresist. With the increase in the exposure time, the width of the pattern initially decreases at a faster rate because of the scattering and 
TABLE 2: Channel width and strength of photoresist obtained by different exposure times.

\begin{tabular}{lccc}
\hline $\begin{array}{l}\text { Experiment } \\
\text { number }\end{array}$ & $\begin{array}{c}\text { Exposure } \\
\text { time }(\mathrm{s})\end{array}$ & $\begin{array}{c}\text { Image width } \\
(\mu \mathrm{m})\end{array}$ & $\begin{array}{c}\text { Photoresist } \\
\text { strength }(\mathrm{N})\end{array}$ \\
\hline 1 & 30 & 120.3 & 18.8 \\
2 & 60 & 116.7 & 19.3 \\
3 & 90 & 108.0 & 19.7 \\
4 & 120 & 99.7 & 20.0 \\
5 & 150 & 98.2 & 20.1 \\
6 & 180 & 97.6 & 20.7 \\
7 & 210 & 96.3 & 21.0 \\
\hline
\end{tabular}

diffraction effect of light. The negative photoresist contains cross-linkers that are activated during exposure. As the exposure time increases, the degree of cross-linking also increases. While developing the substrate, in the case of a lower exposure time, the erosion of photoresist occurs, resulting in a larger pattern width. As the cross-linking of negative photoresist increases with the exposure time, the peel-off resistance of the photoresist also increases. As shown in Figure 4, an exposure time of $120 \mathrm{~s}$ results in a line width of $99.42 \mu \mathrm{m}$ (the desired line width is $100 \mu \mathrm{m}$ ). Figure 4 indicates that a $120 \mathrm{~s}$ exposure time is enough. When the exposure time is less, the photomask pattern does not absorb sufficient UV energy. To estimate the photoresist strength, a scratch test is conducted in which a diamond stylus with a $200 \mu \mathrm{m}$ tip radius is drawn over the sample surface until the coating detaches. As the exposure time increases, the photoresist film becomes harder, so a greater strength is needed to scratch the photoresist further, as shown in Figure 4. The increased exposure time causes an increase in temperature, so the photoresist becomes harder. The effect of the exposure dose is not taken into account because the exposure is performed at a constant light intensity.

\section{Effect of Rolling Direction on Depth of Etch}

Figure 5 shows the effect of the rolling direction on the depth of the etch. From the graph, it is clear that the depth of the etch is increased along the rolling direction and decreases across the rolling direction. This is because of the larger residual stresses present along the rolling direction, as measured on a Bruker EDAx machine. The value of the average residual stress present along the rolling direction is $269.112 \mathrm{Mpa}$, while for across the rolling direction, it is $247.123 \mathrm{MPa}$. If the material is stressed, it will corrode more rapidly, so the etching rate is faster along the rolling direction, leading to a greater depth of etch. A $49.2 \mu \mathrm{m}$ depth is obtained along the rolling direction, while a $42.19 \mu \mathrm{m}$ depth is produced across the rolling direction, as shown in Figures 5 and 6. The rolling direction [001] plane contains more atomic boundaries. As the number of boundaries increases, the chemical attack on the material increases, leading to faster etching and an increased depth of etch. The anisotropic etching also

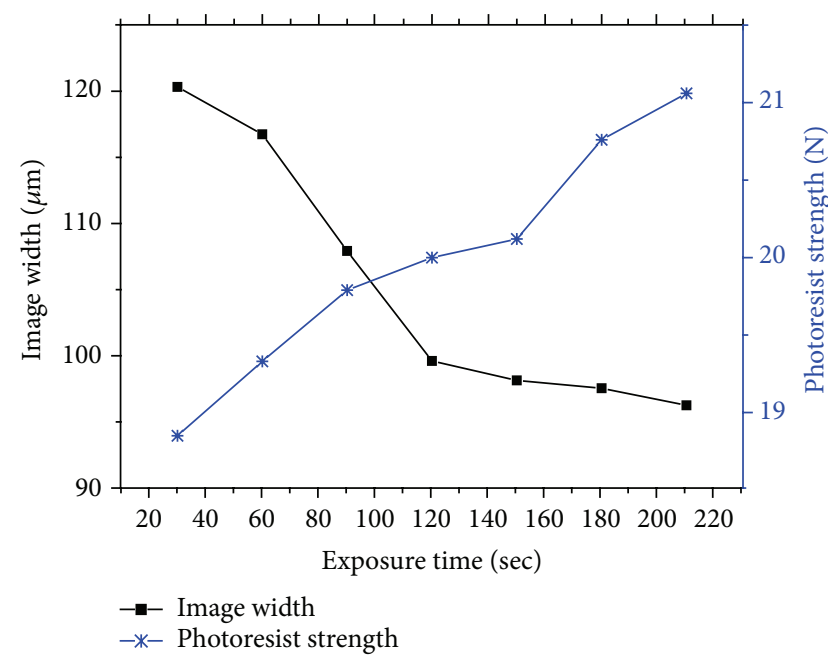

FIGURE 4: Effect of exposure time on image width and photoresist film strength.

improves the channel accuracy. Figure 5 shows that the finish and edge accuracy of the microchannel is better than across the rolling direction.

\section{Effect of Temperature on Depth of Etch and Undercut}

The effect of temperature on the depth of the etch is seen in Figure 7. The measurement of the depth and width after etching is carried out using a white light interferometry instrument (Veeco). It shows that the depth of etch significantly increases with the etching temperature. Upon increasing the temperature from $50^{\circ} \mathrm{C}$ to $70^{\circ} \mathrm{C}$ for a $250 \mu \mathrm{m}$ channel, the depth of etch is approximately 4 and $16.5 \mu \mathrm{m}$, respectively, whereas for a $60 \mu \mathrm{m}$ channel, the depth of etch is $1 \mu \mathrm{m}$ to $10.6 \mu \mathrm{m}$ for the same temperature. As the width of the channel increases, the depth of etch also increases. This difference is because a larger area is exposed in the $250 \mu \mathrm{m}$ channel. According to the Arrhenius equation [25], a high temperature increases the etch rate. An increase in the momentum of molecules at high temperature may also cause an increase in the depth of etch.

The effect of the etching temperature on undercut is studied. It is observed that the undercut is low at $50^{\circ} \mathrm{C}$. Etching at $60^{\circ} \mathrm{C}$ causes a significant increase in the undercut, and the highest increase is observed at $70^{\circ} \mathrm{C}$. The effect of temperature on the undercut of $250,200,150,100$, and $60 \mu \mathrm{m}$ channel widths is shown in Figure 8(a). A Nikon microscope photograph of variable-width etched microchannels is shown in Figure 8(b). The horizontal etching increases as the width of the channel increases, and the channel achieves a semicircular shape, as shown in Figure 8(c). The semicircular shape is useful for maintaining a smooth fluid flow in heat exchangers and in microchannels. If the channels are perfectly flat, there may be a chance of accumulation at the edges and corners, which can result in resistance to mass transfer. 


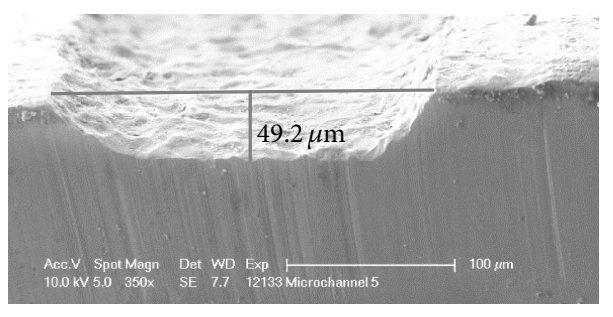

(a) Microchannel depth along rolling direction

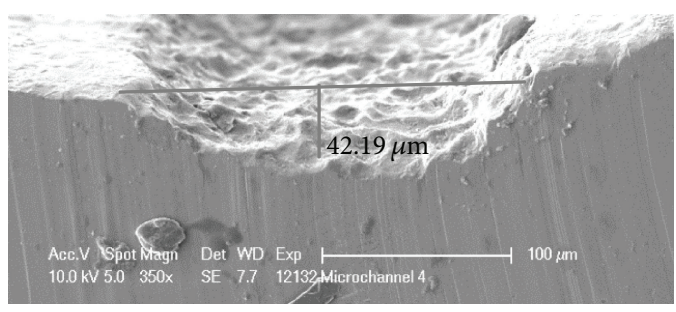

(b) Microchannel depth across rolling direction

Figure 5: Effect of rolling direction on depth of etch.

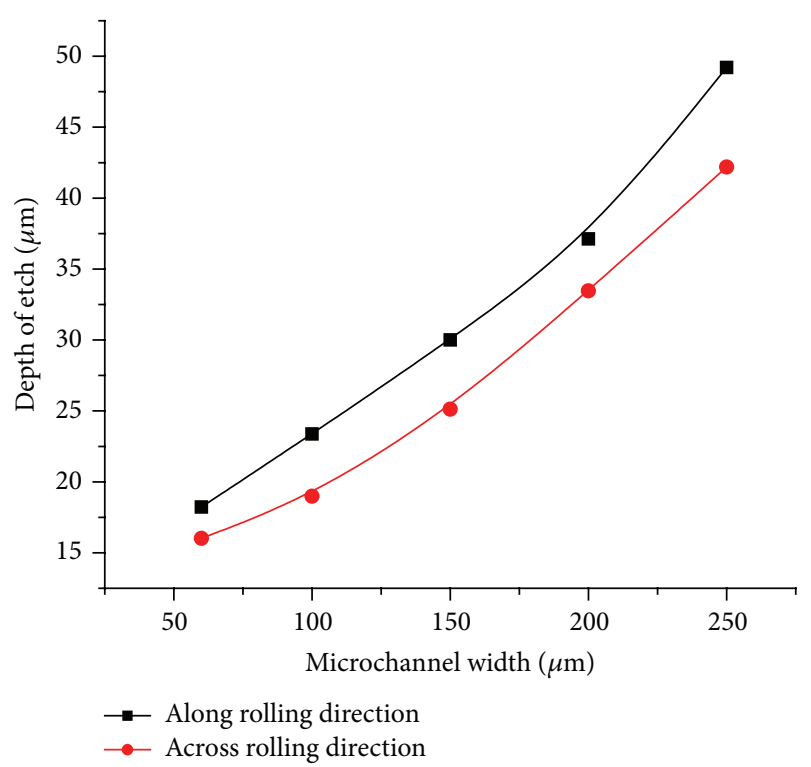

FIGURE 6: Effect of rolling direction on depth of etch.

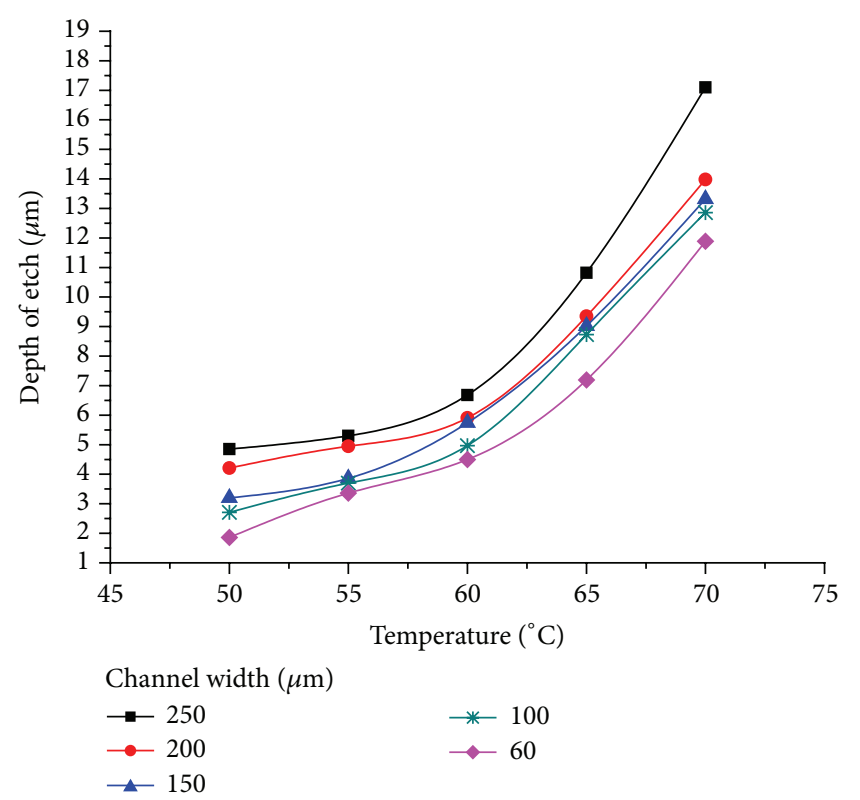

FIgURE 7: Effect of temperature on depth of etch for different channel widths.

\section{Effect of Etching Time on Undercut and Depth of Etch}

It is observed that as time passes, the undercut increases. Figure 9 shows the effect of time on the undercut for different microchannel widths. It also shows that as the time and width of the channels increase, the undercut increases. The initial undercut observed for the $250 \mu \mathrm{m}$ channel was $20 \mu \mathrm{m}$, and for the $60 \mu \mathrm{m}$ channel, the undercut observed was $5 \mu \mathrm{m}$ after $3 \mathrm{~min}$. After $7 \mathrm{~min}$, the undercut was $80 \mu \mathrm{m}$ for the $250 \mu \mathrm{m}$ width and $23 \mu \mathrm{m}$ for the $60 \mu \mathrm{m}$ width. This reveals that the undercut depends on the channel width and time. As the width increases, the undercut increases because there is more horizontal etching across the plane.

The effect of the etching time on the channel depth is studied. The depth of the cavity may be controlled using the etching time and etch rate. The channel depth and width are increased with an increase in etching time. From the plot shown in Figure 10, it is clear that deeper channels are achieved with a higher width. A maximum depth of etch of the $250 \mu \mathrm{m}$ channel width at $7 \mathrm{~min}$ and a minimum depth of etch of the $60 \mu \mathrm{m}$ channel width at $3 \mathrm{~min}$ have been observed.

\section{Conclusions}

In the present study, the effects of the rolling direction and the effects of time, temperature, and concentration on microchannels of Monel 400 were studied. The conclusions from this experimental study are as follows:

(i) An etching mechanism study shows that the rate of reaction of $\mathrm{Cu}$ is faster than that of $\mathrm{Ni}$. $\mathrm{Cu}$ which has a high oxidation potential and high electron mobility towards $\mathrm{Ni}$, which has a smaller oxidation potential, leads to a reaction between $\mathrm{Cu}, \mathrm{Ni}$, and the etchant.

(ii) The photoresist film thickness depends on the spinning speed and spinning time. It is observed that the lower the film thickness, the lower the antietching ability, and vice versa.

(iii) The resolution of the PCM process is mainly affected by the exposure of the coated substrate. A sufficient exposure time and exposure dose are required to develop a fine pattern. During the study, it was found that an exposure time of $120 \mathrm{~s}$ gives the best results for a $100 \mu \mathrm{m}$ line width. The increase in exposure time increases the strength of the photoresist. 


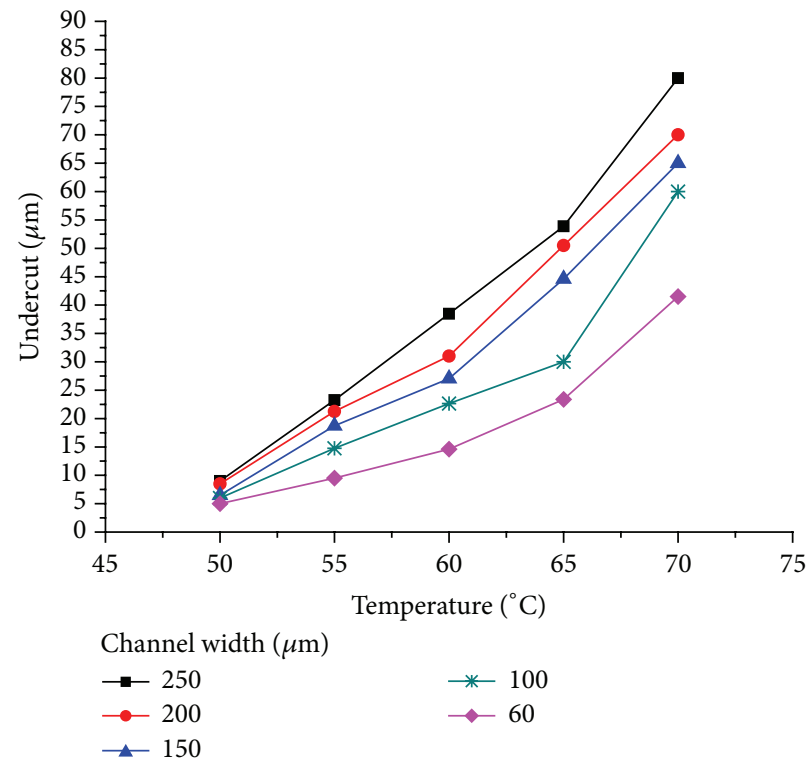

(a)

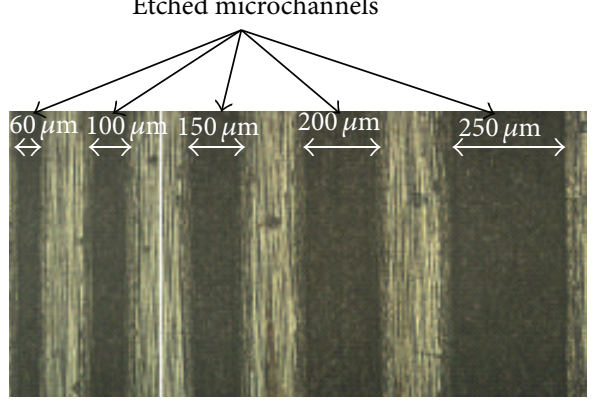

(b)

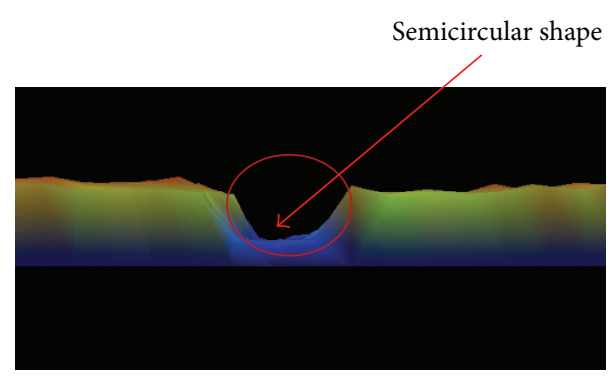

(c)

Figure 8: (a) Effect of temperature on undercut for different channel widths. (b) Nikon microscope image of etched channels. Temperature: $55^{\circ} \mathrm{C}$; etching time: $6 \mathrm{~min}$; and concentration: $600 \mathrm{~g} / \mathrm{L}$. (c) Semicircular shape of microchannel (white light interferometry image).

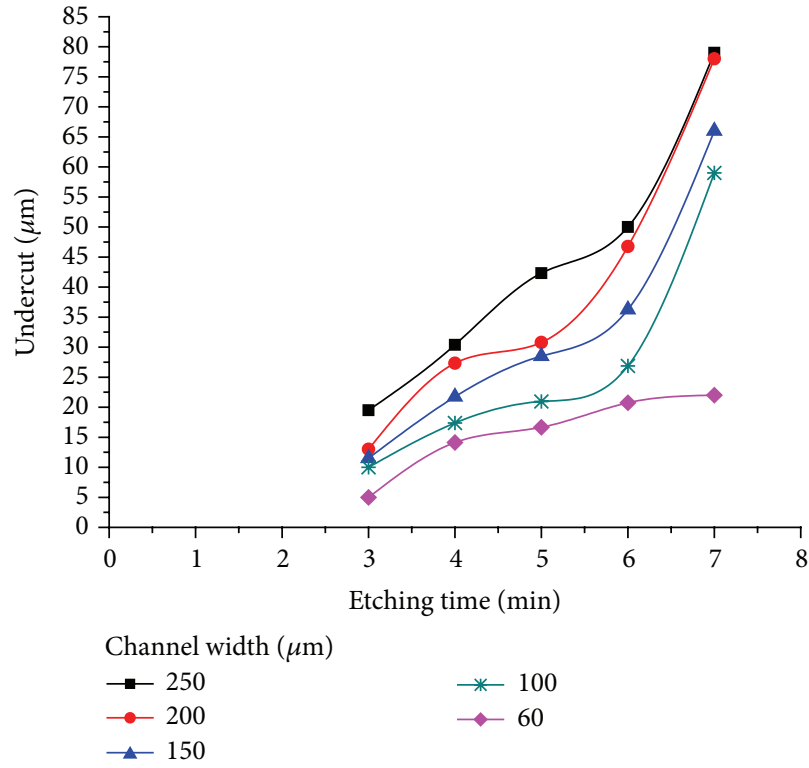

FigURE 9: Effect of etching time on undercut for different channel widths. 


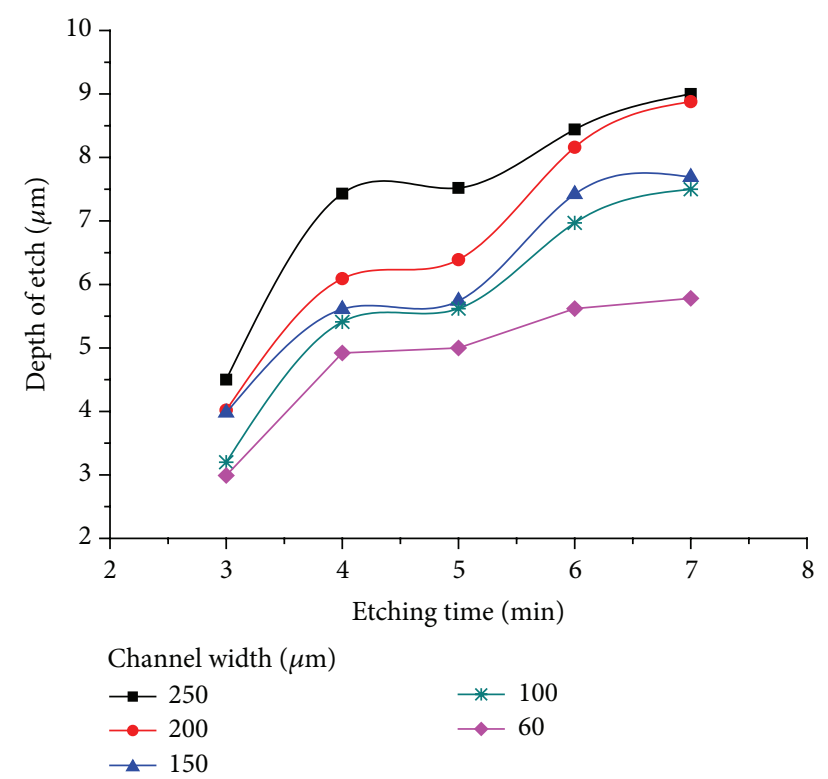

Figure 10: Effect of etching time on depth of etch for different channel widths.

(iv) The depth of etch along the rolling direction is greater than that across the rolling direction. Residual stresses may cause a higher etch rate along the rolling direction compared to across the rolling direction, so that a greater depth of etch is achieved in the along the rolling direction condition.

(v) The channel width and depth are significantly affected by the composition of the etchant, etching time, and temperature. An increased temperature increases the momentum of the molecules, which further causes an increase in the depth of etch and the width of the channels. As the width of the channel increases, the depth of the etch also increases because a larger area is exposed for etching and there is more horizontal etching across the plane. Horizontal etching results in a semicircular-shaped microchannel, which is useful for smooth fluid flow. Depending upon the desired width and depth of the microchannel, the initial width can be optimized.

\section{Competing Interests}

The authors declare that they have no competing interests.

\section{References}

[1] H.-C. Jeong, D.-W. Kim, G.-M. Choi, and D.-J. Kim, "The effect of spray characteristics on the etching of invar alloy with $\mathrm{FeCl}_{3}$ solution," International Journal of Precision Engineering and Manufacturing, vol. 10, no. 4, pp. 107-114, 2009.

[2] H.-P. Tsui, J.-C. Hung, K.-L. Wu, J.-C. You, and B.-H. Yan, "Fabrication of a microtool in electrophoretic deposition for electrochemical microdrilling and in situ micropolishing," Materials and Manufacturing Processes, vol. 26, no. 5, pp. 740745, 2011.
[3] R. Arunachalam and M. A. Mannan, "Machinability of nickelbased high temperature alloys," Machining Science and Technology, vol. 4, no. 1, pp. 127-168, 2000.

[4] S. P. Leo Kumar, J. Jerald, S. Kumanan, and R. Prabakaran, "A review on current research aspects in tool-based micromachining processes," Materials and Manufacturing Processes, vol. 29, no. 11-12, pp. 1291-1337, 2014.

[5] M. Malapati and B. Bhattacharyya, "Investigation into electrochemical micromachining process during micro-channel generation," Materials and Manufacturing Processes, vol. 26, no. 8, pp. 1019-1027, 2011.

[6] W. Ehrfeld, V. Hessel, and H. Lowe, Micro Reactors, Wiley-VCH, Weinheim, Germany, 2000.

[7] Z. He, D. Li, L. Cao, and Y. Tang, "Fabrication and characterization of cross-connected microchannel porous mesh plates (CCMPMPs) by multi-cutter milling," Materials and Manufacturing Processes, vol. 30, no. 2, pp. 256-262, 2015.

[8] O. Çakır, "Chemical etching of aluminium," Journal of Materials Processing Technology, vol. 199, no. 1-3, pp. 337-340, 2008.

[9] C. Ha and N.-J. Park, "Effect of rolling direction on the development of microstructure, texture, and mechanical properties of AZ31B alloys," International Journal of Precision Engineering and Manufacturing, vol. 15, no. 9, pp. 1955-1959, 2014.

[10] I. Topic, H. W. Höppel, and M. Göken, "Influence of rolling direction on strength and ductility of aluminium and aluminium alloys produced by accumulative roll bonding," Journal of Materials Science, vol. 43, no. 23-24, pp. 7320-7325, 2008.

[11] Y. Park, J.-H. Kang, and Y.-K. Lee, "The relationship of microstructure and texture to rolling direction in cold-rolled metastable austenitic steel," Metallurgical and Materials Transactions A: Physical Metallurgy and Materials Science, vol. 42, no. 3, pp. 692-699, 2011.

[12] N. S. Kogut, "Effect of the direction of rolling on crack resistance of bridge steels," Problemy Prochnosti, no. 2, pp. 9-12, 1984.

[13] A. I. Asphahani, "Corrosion of nickel base alloys," in ASM Handbook, vol. 13a, ASM International, 2005.

[14] J. R. Crum, Major Application and Corrosion Performance of Nickel Alloys, 9th Edition of ASM Metal Handbook, Inconel Alloys International Inc, 1987.

[15] R. Simpson and S. Almonacid, "Plate heat exchangers," in Encyclopedia of Agricultural, Food, and Biological Engineering, pp. 763-766, 2007.

[16] E. A. Tice, "Effects of air pollution on the atmospheric corrosion behavior of some metals and alloys," Journal of the Air Pollution Control Association, vol. 12, no. 12, pp. 553-559, 1962.

[17] Y.-K. Yang and T.-C. Chang, "Experimental analysis and optimization of a photo resist coating process for photolithography in wafer fabrication," Microelectronics Journal, vol. 37, no. 8, pp. 746-751, 2006.

[18] V. G. Kutchoukov, J. R. Mollinger, and A. Bossche, "New photoresist coating method for 3-D structured wafers," Sensors and Actuators, A: Physical, vol. 85, no. 1, pp. 377-383, 2000.

[19] Z. W. Zhong, X. C. Shan, and Y. C. Yao, "Investigation of antiadhesive coatings for nanoimprinting lithography," Materials and Manufacturing Processes, vol. 25, no. 7, pp. 658-664, 2010.

[20] C. Wang, S.-J. Cho, and N.-Y. Kim, "SU-8-based structural material for microelectronic processing applications," Materials and Manufacturing Processes, vol. 28, no. 8, pp. 947-952, 2013.

[21] A. Narasimhan, "Thermal analysis of a silicon wafer processing combination bake-chill station used in microlithography," Materials and Manufacturing Processes, vol. 20, no. 2, pp. 273286, 2005. 
[22] J. M. del Hoyo-Meléndez and M. F. Mecklenburg, "An investigation of the reciprocity principle of light exposures using microfading spectrometry," Spectroscopy Letters, vol. 44, no. 1, pp. 52-62, 2011.

[23] J. Zhang and Y. Meng, "A study of surface texturing of carbon steel by photochemical machining," Journal of Materials Processing Technology, vol. 212, no. 10, pp. 2133-2140, 2012.

[24] H. H. Uhlig, Corrosion and Corrosion Control, chapter 22, John Wiley and Sons, 1963.

[25] R. Ueda, "Chemical machining with a ferric chloride etchant," Corrosion Engineering, vol. 38, no. 4, pp. 271-282, 1989. 

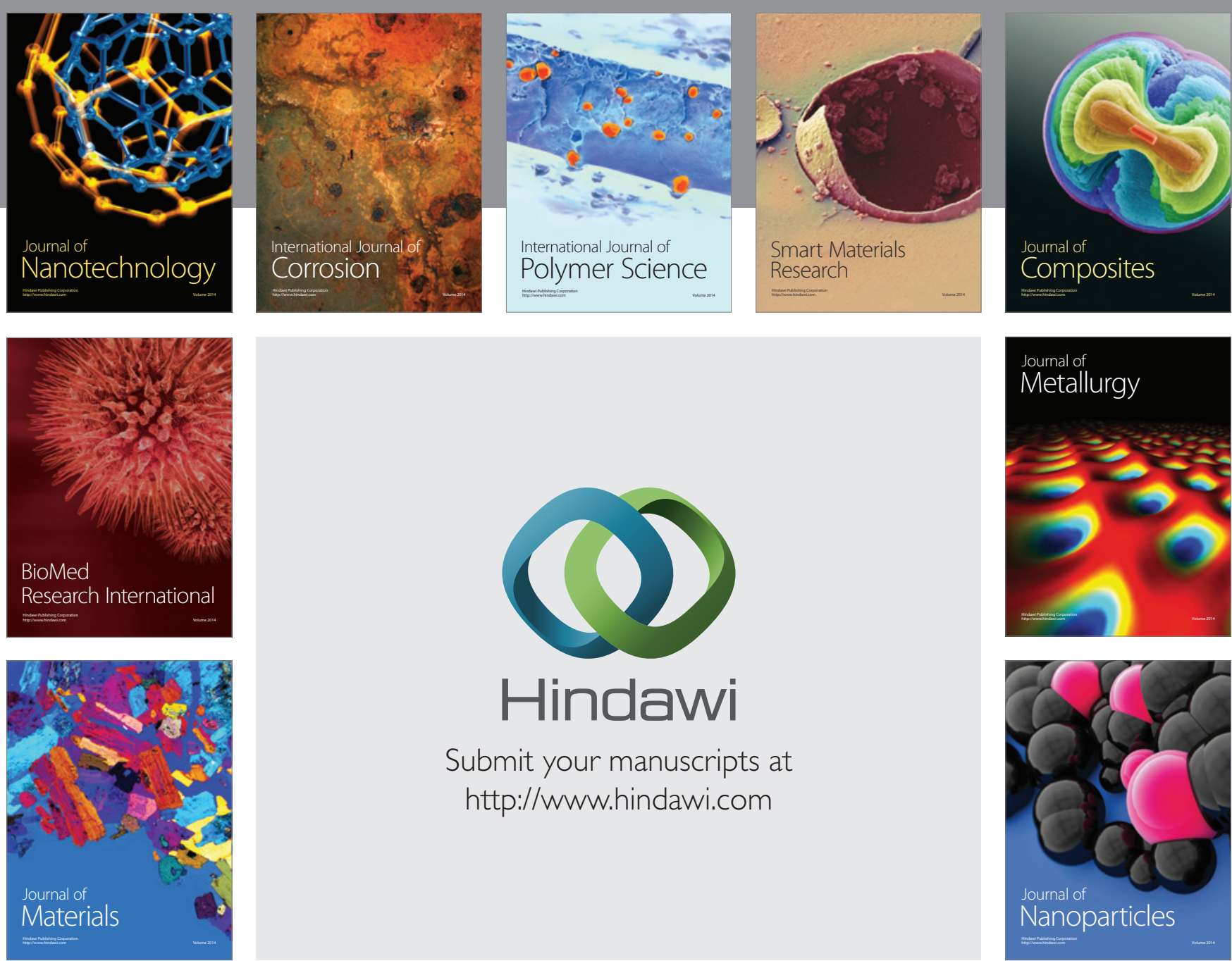

\section{Hindawi}

Submit your manuscripts at

http://www.hindawi.com

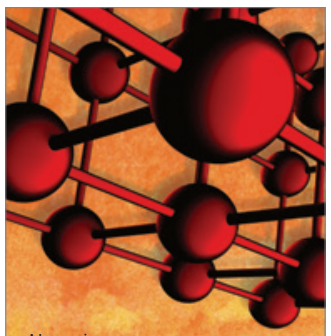

Materials Science and Engineering
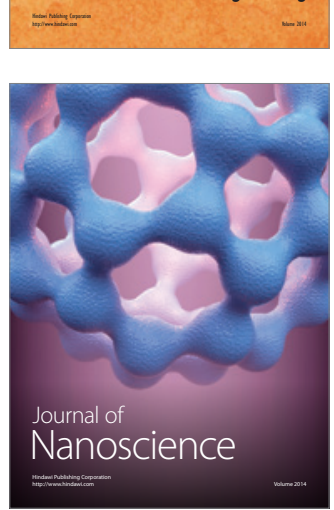
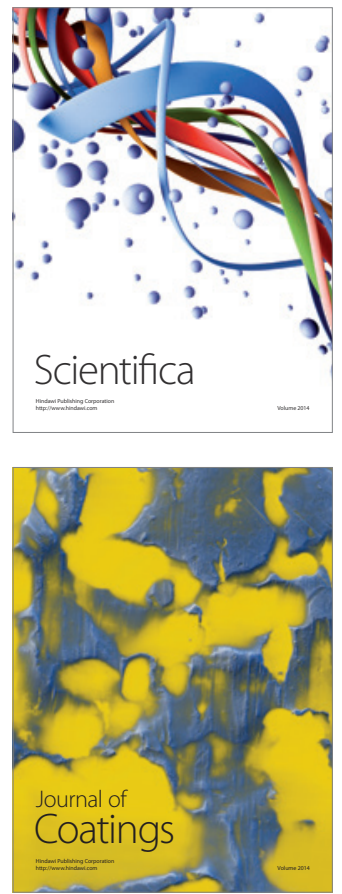
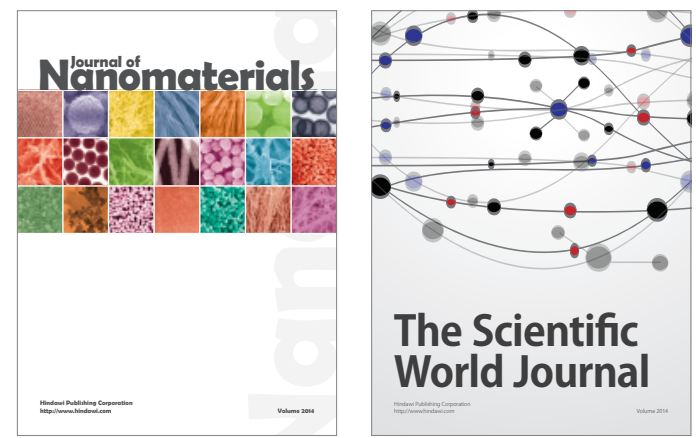

The Scientific World Journal
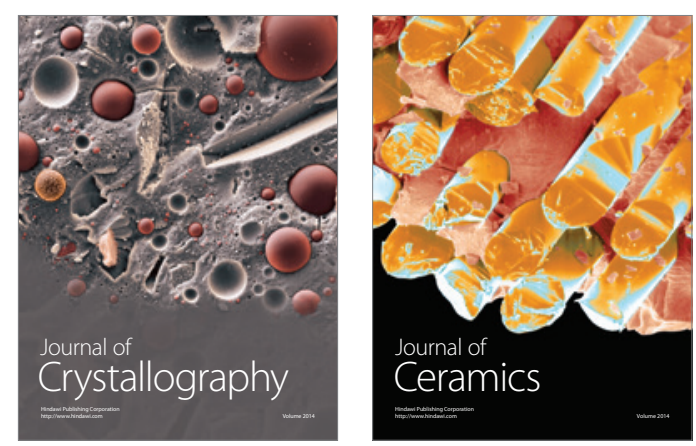
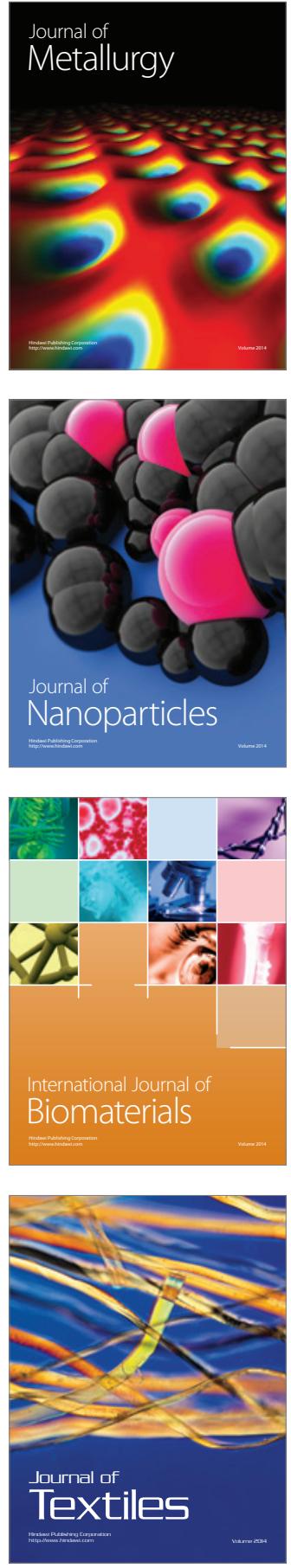